\title{
Strates
}

STRATES Matériaux pour la recherche en sciences sociales

$12 \mid 2006$

Nouvelles tensions impériales et recompositions en Europe centrale, orientale, et CEI

\section{La transition monétaire en Europe centrale : fondements théoriques}

\section{Olivier STINTZY}

\section{(2) OpenEdition}

\section{Journals}

Édition électronique

URL : http://journals.openedition.org/strates/1962

DOI : $10.4000 /$ strates. 1962

ISSN : $1777-5442$

Éditeur

Laboratoire Ladyss

Édition imprimée

Date de publication : 31 décembre 2006

ISSN : 0768-8067

Référence électronique

Olivier STINTZY, «La transition monétaire en Europe centrale : fondements théoriques », Strates [En ligne], 12 | 2006, mis en ligne le 20 juillet 2007, consulté le 08 septembre 2020. URL : http:// journals.openedition.org/strates/1962 ; DOI : https://doi.org/10.4000/strates.1962

Ce document a été généré automatiquement le 8 septembre 2020.

Tous droits réservés 


\title{
La transition monétaire en Europe centrale : fondements théoriques ${ }^{1}$
}

\author{
Olivier STINTZY
}

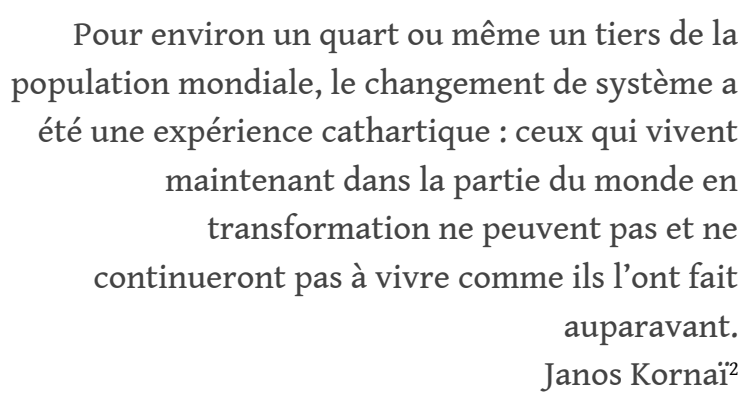

1 Le système de planification économique en vigueur dans les pays d'Europe centrale avant la transition comportait son propre mécanisme de coordination - largement sous-optimal - dont la monnaie était quasiment absente, ce qui permet de le qualifier d'a-monétaire ${ }^{3}$. Il s'effondra avant que le nouveau mécanisme de coordination par le marché ne s'instaure avec la transition: celle-ci ne peut donc pas recouvrir la simple "mutation" de normes socialistes existantes en nouvelles normes capitalistes mais bien la construction d'un nouveau système économique en réponse à une situation que Janos Kornaï (2000) qualifie de no-man's land institutionnel. Le processus de transition sera donc appréhendé ici comme la construction des «institutions mutuellement interdépendantes" (Aoki, 20014) composant le système d'économie de marché, au premier rang desquelles la monnaie.

2 En effet, des analyses récentes (Aglietta et Cartelier, 1998; Aglietta et Orléan, 2002) ont mis en lumière le fait que, dans une économie de marché - caractérisée par des échanges interdépendants mais décentralisés dans le temps et dans l'espace - le mécanisme de coordination fondamental est la monnaie, institution sociale et système de paiement permettant la coordination des actions des agents : le système d'économie planifiée ayant été considéré comme a-monétaire, la transition vers l'économie de marché se présente alors comme un processus intrinsèquement institutionnel d'« affirmation de l'économie monétaire » (Zlotowski, 1998). 
3 Ainsi, la présente recherche retiendra l'institutionnalisation de la monnaie ${ }^{5}$ comme grille de lecture du processus de transition économique, appréhendé comme la construction de systèmes monétaires (et bancaires) au sein des économies en transition d'Europe centrale. En cela, cette approche cherche à contribuer à une tentative nécessaire - de réintégration de la monnaie dans le corpus des théories institutionnelles, dans la perspective d'une analyse du processus transformationnel des économies postcommunistes.

Contrainte monétaire lâche et mode de régulation des économies planifiées

4 J. Kornaï (1984) a analysé le système d'économie socialiste comme une «économie de la pénurie » dans lequel la monnaie ne joue qu'un rôle passif dans la sphère de la production. Pour Laurence Scialom et Yves Zlotowski (1994), le système des anciennes économies planifiées était organisé autour d'une contrainte budgétaire lâche s'apparentant à un ensemble de procédures de régulation basé sur des corpus de règles explicites et implicites connues de tous les agents («common knowledge ») ${ }^{6}$. Celles-ci permettaient aux entreprises d'assouplir leur contrainte budgétaire en obtenant des crédits hors-plan auprès de la monobanque puis de recourir au crédit inter-entreprises, lorsque la Banque centrale opérait un arrêt brutal de la distribution du crédit afin de stopper le dérapage monétaire, la Banque centrale régularisant ces opérations au début de chaque nouveau Plan ${ }^{7}$. Le système d'économie planifiée apparaît donc comme « un système extrêmement décentralisé d'émission monétaire, où l'initiative du crédit revient aux entreprises » (Benoist, 1994) et où la relation de paiement s'apparente à un "octroi de crédit forcé » (Sapir, 1989), le groupe de la monobanque n'exerçant aucun contrôle sur la contrainte monétaire des entreprises. La monnaie ici n'est qu'un support aux échanges mais ne peut en aucun cas jouer le rôle central de coordination propre à l'économie de marché : la seule contrainte budgétaire est celle du règlement des soldes en fin de cycle, qui ne s'applique qu'à la Banque centrale: le prêteur en dernier ressort est ici fortement contraint, mais uniquement dans un certain volume dont il a le contrôle. La différence première entre le système d'économie planifiée et celui d'économie de marché réside ainsi avant tout dans le rôle joué par la monnaie, ce qui permet d'analyser la transition sous l'angle de la mise en place du processus de coordination des actions des agents par la monnaie.

5 Largement sous-optimal, ce mécanisme de coordination s'effondra en grande partie avant le commencement du processus de transition et l'instauration progressive d'un nouveau système de coordination par le marché. La transition ne recouvre donc pas la transformation de normes socialistes existantes en de nouvelles normes "libérales " mais doit être appréhendée comme la réponse à une situation d'anomie (Blanchard, 1997) que J. Kornaï (2000) qualifie de «no-man's land institutionnel». Compte tenu du rôle de coordination assurée par la monnaie au sein d'une économie de marché, le processus de transition économique en Europe centrale peut ainsi être appréhendé comme la construction de systèmes monétaires.

Institution monétaire et système de paiement en économie de marché

6 La théorie générale opérant une distinction artificielle entre l'établissement de l'accord entre les agents (situation d'équilibre) et leur action effective sur le marché par la réalisation des échanges, ces derniers ne peuvent être effectués qu'à l'équilibre et les agents ont la certitude de respecter leur contrainte budgétaire ${ }^{8}$. Retenir le principe plus réaliste de décentralisation des échanges, conduit à renouer l'établissement d'un accord (par la formation d'un prix) à la réalisation effective de l'échange pour que la 
transaction puisse avoir lieu. Dès lors, le marché doit être considéré comme un instrument de coordination a posteriori: en d'autres termes, ce n'est que par leur participation au marché que les agents peuvent connaître le degré de réalisation de leurs actions'. Si l'on accepte la réalisation des transactions de manière décentralisée et donc potentiellement en dehors de la contrainte budgétaire des agents économiques, il faut fournir aux agents le moyen de leur exécution ${ }^{10}$ : c'est le rôle de la monnaie comme moyen général de paiement, qui apparaît dès lors comme «ce mode d'accord particulier, spécifique de la coordination par le marché » (Cartelier, 1991).

7 Ces développements s'inscrivent dans la perspective du profond renouvellement de la conception de la monnaie introduit par les travaux de Joseph M. Ostroy et Ross M. Starr (1990) pour qui la double décentralisation géographique et temporelle du marché implique d'abandonner l'hypothèse d'une information parfaite au profit de celle d'une information imparfaite, chaque agent ne connaissant ici que sa propre contrainte budgétaire en l'absence du commissaire priseur, centralisateur de l'information. Les auteurs plaident ainsi pour l'introduction d'une autorité extérieure chargée de collecter, synthétiser et conserver l'information sur les déséquilibres issus des échanges ${ }^{11}$ : dans un univers où l'information détenue par les agents est imparfaite, la fonction de dispositif de mémorisation (record keeping device) proposée par les auteurs doit être considérée comme la fonction essentielle de la monnaie. Celle-ci permet de connaître les scores économiques du passé d'échangiste du payeur ou la validité de ses projets économiques ${ }^{12}$. Dès lors, la monnaie n'est pas seulement un moyen de conserver l'information, elle est aussi un moyen de la faire circuler et remplace donc le commissaire-priseur walrassien dans sa fonction d'«outil d'imposition de la contrainte budgétaire » (Zlotowsky, 1998).

8 Les implications d'un tel raisonnement sont primordiales car elles placent la monnaie au cœur de l'échange marchand: par cette procédure, les agents économiques « constatent leur accord et peuvent l'opposer à autrui c'est-à-dire le traduire en un fait social constatable par quiconque ${ }^{13} »$. Pour Michel Aglietta et Jean Cartelier :

9 - la monnaie comme institution doit être le point de départ de la démarche économique car seule, semble-t-il, elle permet de rendre compte de la combinaison particulière des principes de décentralisation et d'interdépendance (Aglietta, Cartelier, 1998) ;

10 - la monnaie doit donc bien être considérée comme une institution économique placée au cœur du système d'économie de marché. Dans cette optique, la monnaie-institution doit être comprise au sens large de son acception, à savoir le système de paiement, tel que décrit par ces auteurs :

Par "théorie économique de la monnaie », il faut entendre l'énoncé des propriétés minimales que l'institution «monnaie » doit posséder pour rendre compte de la coordination des actions privés par le marché. Dans cette perspective, la monnaie [...] est un système de paiement défini par un ensemble minimal de règles à partir duquel il est possible de décrire un maximum de situations possibles (Aglietta, Cartelier, 1998).

11 Ces développements peuvent être rapprochés du corpus théorique des nouvelles théories institutionnelles - et en particulier $d u$ cadre d'analyse proposé très récemment par Masahiko Aoki (2001) - ce qui permet de donner une lecture institutionnelle du processus de transition en Europe centrale.

Les nouvelles théories institutionnelles et la monnaie 
12 Depuis Adam Smith, l'économie a été assimilée à un jeu (« the great chessboard of human society ${ }^{14}$ » [Le grand échiquier de la société humaine]), situation dans laquelle les agents individuels interagissent les uns avec les autres en fonction de leurs motivations personnelles. En utilisant cette analogie, trois conceptions distinctes des institutions peuvent être isolées (Aoki, 2001). La conception habituelle (non économique) de l'institution l'assimilant aux participants au jeu (players of the game) ne fait pas de distinction entre le jeu et les joueurs qui y participent (les gouvernements, les associations, «l'institution judiciaire», etc.) (Nelson, 1994, p. 47-63). Douglass North (1997) a été parmi les premiers à s'opposer à cette conception en assimilant l'institution aux règles du jeu alors que les joueurs en sont les organisations, à savoir des groupes d'individus engagés dans une action intentionnelle (purposive action) : les institutions apparaissent alors comme des contraintes humainement imposées qui structurent les interactions humaines fondant dans le temps la performance des sociétés et des économies (North, 1994). La troisième conception des institutions les analyse en termes d'équilibre du jeu : c'est dans cette perspective que s'inscrivent les nouvelles théories institutionnelles, ainsi que la conception de Avner Greif (1994) décrivant les institutions comme composées de règles complétées par des organisations.

Le questionnement de $\mathrm{M}$. Aoki consiste à comprendre comment une contrainte particulière (parmi les menus de choix respectifs) est collectivement perçue par l'ensemble des joueurs comme menant à l'équilibre et cela de manière répétitive. L'auteur y répond en donnant sa propre définition d'une institution qu'il considère comme « un système auto-entretenu de croyances communes quand à un déroulement précis et répété du jeu ${ }^{15}$ ». Un "déroulement précis et répété du jeu » est considéré comme une "règle du jeu ", ce qui rapproche cette définition de celle de D. North, mais ici la "règle du jeu" est d'origine endogène car créée par l'interaction stratégique des agents et en permanence présente à leur esprit. L'apport de M. Aoki réside principalement dans son interprétation du système de croyances communes. Même s'il considère, comme A. Greif, l'existence d'une institution comme reflétant les croyances des agents, celles-ci doivent acquérir, selon lui, des «bases substantielles » d'existence afin d'être partagées par eux - de manière persistante et endogène - et considérées comme appropriées pour déterminer les conséquences de leurs choix. Ces croyances partagées sont constituées de représentations sommaires (summary representation) i.e. de condensés d'informations (compressed information) sur un équilibre du jeu parmi l'ensemble des équilibres théoriques ${ }^{16}$. La référence à la notion de condensés d'informations mérite de plus amples précisions. L'auteur suppose ici que le jeu étant répété à de nombreuses reprises, un résultat stable (stable outcome) est régulièrement atteint et que progressivement chaque agent, en se basant sur sa propre expérience, acquiert une idée « raisonnable » du déroulement du jeu. Cette perception fait appel à une rationalité limitée, les agents ne connaissant que certains faits caractéristiques concernant les règles de conduite supposées des autres agents, mais ce condensé d'informations est supposé suffisant pour leur permettre d'anticiper le déroulement du $\mathrm{jeu}^{17}$.

14 Cette conception de "condensés d'informations" se rapproche des travaux de la théorie des conventions, pour laquelle les conventions résument du savoir et permettent ainsi à des agents l'ignorant d'adopter les comportements qu'ils suivraient s'ils le possédaient (Favereau, 1989) : ces «condensés d'informations » permettent aux joueurs de former leurs stratégies d'action personnelles en réponse à l'évolution de 
l'environnement, en se basant sur leurs seules perceptions subjectives (leurs " croyances») des stratégies des autres joueurs. C'est uniquement lorsque leurs perceptions sont stabilisées (et donc répétitives) que les agents peuvent définir leurs propres règles d'actions qui deviennent à leur tour des indications pour le déroulement $\mathrm{du}$ jeu. Ce que $\mathrm{M}$. Aoki présente comme un phénomène de rétro-action (complex feedback mechanism) s'apparente à la notion d'interdépendance entre les joueurs, qui est une des caractéristiques principales de l'économie de marché. M. Aoki assimile cette caractéristique de persistance évoluant en fonction des perceptions subjectives des agents et de leurs stratégies de décision des règles du jeu à un équilibre de Nash, s'inscrivant bien en cela dans la perspective de l'institution conçue comme un équilibre du jeu: les institutions peuvent ainsi être caractérisées de "parties des croyances des agents - relatives à l'équilibre - communes à (presque) tous, et concernant la méthode de déroulement du jeu ${ }^{18}$ ». L'auteur insiste sur le fait que les institutions ne sont pas le fruit d'anticipations parfaitement rationnelles dans le cadre d'un jeu se déroulant sur une seule période. Au contraire, la construction des institutions s'inscrit dans un temps long nécessaire à la stabilisation des perceptions subjectives de chacun des acteurs et à leur rétroaction sur l'ensemble du jeu, comme le souligne David M. Kreps: "An institution is the product of long term experiences of a society of boundedly rational and retrospective individuals ${ }^{19}$.»

15 La conception des institutions que donne M. Aoki permet de rendre totalement endogène la formation de l'équilibre mais aussi de montrer que le choix d'une stratégie d'action est le fruit d'un long "apprentissage » fondé sur l'expérience des joueurs : l'équilibre est ici appréhendé comme une "réalité socialement construite » [a socially constructed reality] totalement endogène. Le phénomène se renforce de lui-même, à mesure que les agents - dont les "croyances» dirigent les choix stratégiques reproduisent la situation d'équilibre et que celle-ci, en retour, reconfirme le " condensé d'informations » qu'elle représente pour les joueurs. Pour M. Aoki, c'est en cela que l'institution peut devenir une "réalité sociale", en dépit de son origine purement endogène.

Cette définition met ainsi en lumière le caractère intrinsèquement cognitif de l'institution: ce sont les "croyances partagées" des agents qui servent à coordonner leurs actions. Cette approche nous semble particulièrement adaptée à l'analyse du processus de transition : en effet, la dimension cognitive est au cœur de l'approche de la transition donnée par F. Renversez $(2001)^{20}-$ «la série des séquences successives du passage entre deux structures organisant le comportement des agents »-car, dans une approche institutionnelle, ce sont bien les croyances des acteurs économiques qui déterminent leur comportement ${ }^{21}$.

Concevoir la transition comme une "métamorphose institutionnelle" permet d'appréhender celle-ci comme une succession de périodes, phases ou séquences correspondant à la notion d'évolution institutionnelle que M. Aoki (2001) définit comme un "équilibre ponctué", caractérisé à la fois par l'effet de sentier et l'innovation, des points de résistance et de basculement ${ }^{22}$. Nous faisons l'hypothèse que la transition peut être appréhendée comme la succession de différentes séquences correspondant chacune à une situation d'équilibre temporaire (ou « ponctué »), c'est-àdire au résultat d'un processus d'institutionnalisation. L'articulation entre les différentes séquences ou périodes qui rythment le processus de transition correspond à des situations d'évolution institutionnelle caractérisées par un déséquilibre cognitif. 
Lorsque sous l'effet d'une modification de l'environnement, l'institution existante s'avère incapable de fournir une "représentation sommaire " aux agents, ceux-ci interrogent la pertinence et l'utilité de leurs propres «croyances »: le «déséquilibre cognitif global » qui en découle correspond à une crise institutionnelle.

Le rapprochement entre ces développements qui se placent dans le cadre des nouvelles théories institutionnelles, d'une part, et les récentes analyses théoriques de la monnaie présentées supra, d'autre part, nous paraît ainsi particulièrement enrichissant: les développements précédents ont permis d'appréhender la transition économique comme la construction de nouveaux systèmes monétaires et bancaires en réponse à une situation de "no-man's land institutionnel» (Kornaï, 1984). Si la monnaie est le mode de socialisation des agents dans un système d'économie de marché, le processus de transition entre une économie planifiée et un tel système peut être fondamentalement considéré comme un processus de construction de l'institution monétaire, c'est-à-dire d'institutionnalisation de la monnaie ce qui recouvre un long processus de stabilisation des croyances des agents sur un même résultat du jeu (Aoki, 2001), à savoir la coordination de leurs actions par la monnaie. En référence à nos précédents développements, le rôle de l'institution monétaire sera ainsi d'assurer la socialisation des acteurs au sein d'une communauté de paiement caractérisée par une décentralisation temporelle et spatiale des échanges et une incomplétude des marchés.

La transition économique dans les économies postcommunistes recouvre ainsi un processus "ponctué » de construction institutionnelle de nouveaux systèmes monétaires (et bancaires) dont la cohérence est assurée par la stabilisation des "croyances» des agents qui sera analysée comme leur degré de confiance dans la monnaie; la crédibilité des réformes mises en place par les autorités monétaires est donc cruciale pour la réussite $\mathrm{du}$ processus de transition. Le processus d'institutionnalisation de la monnaie se traduit concrètement par l'inscription d'un double ancrage institutionnel de la monnaie, l'un relatif à la politique monétaire, l'autre au système bancaire, qui place la Banque centrale au cœur du dispositif monétaire.

Le Banquier central au cœur du processus d'institutionnalisation de la monnaie

Comme cela a été décrit supra, le système d'économie planifiée qui prévalut en Europe centrale pendant près d'un demi-siècle étant caractérisé par une contrainte budgétaire lâche, le passage à l'économie de marché peut être appréhendé comme l'introduction d'une « contrainte budgétaire dure ». Pour que la monnaie puisse pleinement assumer sa fonction de coordination, sa valeur doit présenter un certain degré de stabilité : la transition institutionnelle peut ainsi être assimilée à l'ancrage de la monnaie qui stabilise sa valeur et permet d'abolir les circuits monétaires multiples.

21 Dans cette perspective, les évolutions entre les différents "dispositifs monétaires" peuvent être appréhendées sous l'angle de la crédibilité de la politique monétaire, partie intégrante de son caractère institutionnel. Les différents dispositifs monétaires adoptés par les autorités en République tchèque, Pologne et Hongrie au cours de la dernière décennie constituent ainsi les principales "séquences» du processus de transition.

22 Les premières années du processus de transition (1989 à 1991-1992) ont ainsi été marquées par une politique de rigueur visant à stabiliser les "fondamentaux économiques "-au premier rang desquels l'inflation - et à restaurer la stabilité des 
monnaies nationales par des stratégies d'ancrages fixes destinées à assurer la crédibilité du change. Le passage d'une politique de stabilisation à une politique de relance dans une seconde séquence du processus de transition (1991-1992 à 1994) a vu l'adoption progressive d'instruments indirects de politique monétaire dont l'utilisation traduit la crédibilisation progressive de la politique monétaire conduite par les autorités : en effet, celles-ci peuvent désormais faire appel à des techniques de marché (opérations d'open-market) plus élaborées mais nécessitant un certaine stabilisation des anticipations (ou "croyances") de l'ensemble des acteurs (autorités monétaires et acteurs privés) que nous analysons comme le résultat d'un processus d'institutionnalisation de la politique monétaire.

Une troisième "période » (1994-1997) a vu la nécessaire ouverture internationale des économies tchèque, polonaise et hongroise, conduisant à de délicats arbitrages entre la stabilité interne de la monnaie (maîtrise de l'inflation) et l'équilibre des comptes extérieurs (préservation de la compétitivité du secteur productif). Pendant cette période, la variable d'ajustement de référence est le taux de change réel, dont l'ancrage est soumis à des anticipations parfois contradictoires de l'ensemble des acteurs d'une économie ouverte (autorités monétaires, secteur privé financier et non-financier, domestique et étranger).

Les stratégies de sortie d'ancrage (quatrième séquence : 1995-1997) témoignent ainsi de la qualité de la stabilisation des croyances de l'ensemble de ces acteurs, c'est-à-dire du degré d'institutionnalisation de la monnaie. Ainsi, les crises monétaires qu'ont connues les économies en transition d'Europe centrale (principalement la crise de change de la couronne tchèque en mai 1997, mais aussi les attaques spéculatives contre le forint hongrois au début de l'année 2003) peuvent être analysées comme des crises de vulnérabilité, déconnectées des fondamentaux économiques et traduisant la défiance des agents vis-à-vis de la monnaie : en cela, une crise monétaire est intrinsèquement une crise institutionnelle témoignant d'un déséquilibre cognitif et sa résolution appelle une évolution institutionnelle se traduisant par une modification de l'ancrage de la monnaie : la République tchèque a ainsi abandonné l'ancrage fixe du change au plus fort de la crise de mai 1997 pour adopter le flottement géré de la couronne.

Les évolutions plus ou moins heurtées de la politique monétaire peuvent ainsi être considérées comme formant une succession d'arrangements institutionnels visant à l'instauration de la confiance dans la monnaie, c'est-à-dire son «ancrage institutionnel » dans les croyances des agents en sa qualité de dispositif central de coordination de leurs actions. À cet égard, le dispositif de ciblage de l'inflation - qui requiert l'indépendance de la Banque centrale - nous apparaît comme l'ancrage monétaire le plus achevé dans la mesure où il se fonde sur l'instauration de la confiance entre les autorités monétaires et les acteurs du marché : il correspond à une cinquième séquence (1997-) du processus de transition dans laquelle les économies tchèque, polonaise et hongroise se situent encore aujourd'hui.

Le tableau synoptique ( $c f$. fin d'article) présente, pour chacune de ces cinq périodes, le contexte macro-économique des trois pays, les objectifs poursuivis par leurs autorités monétaires respectives, les différentes stratégies d'ancrage adoptées, les outils de politique monétaire mis en œuvre et enfin les résultats obtenus ainsi que les éventuels obstacles rencontrés dans la mise en place des différentes politiques monétaires en République tchèque, Pologne et Hongrie. 

planification de l'utilisation des ressources au profit de leur affectation aux emplois les plus rentables économiquement et socialement: au-delà de sa fonction de vecteur d'intégration des sphères monétaire et réelle, le système bancaire sera ainsi considéré comme l'élément indispensable pour assurer le financement du secteur productif, en l'absence de marché financier efficient. La théorie de la banque rappelle néanmoins que les spécificités de l'industrie bancaire en font une activité particulièrement risquée : en raison des importantes dissonances cognitives qu'il véhicule, le processus de transition agit comme un révélateur du risque inhérent au secteur bancaire. Dès lors, le rôle central joué par les banques dans la gestion de la monnaie - mécanisme de coordination sociale par excellence dans une économie de marché - justifie l'existence d'un ensemble de règles prudentielles applicables aux banques. Celui-ci comprend des mesures préventives au niveau microéconomique visant à restreindre ex-ante les prises de risque excessives de la part des établissements de crédit mais aussi des dispositifs agissant au niveau macroéconomiques afin de prévenir les enchaînements pouvant transformer une vulnérabilité bancaire individuelle en une crise de nature systémique.

Les phénomènes de globalisation, libéralisation et d'innovation financières font apparaitre des risques de types nouveaux qui requièrent d'importantes modifications des dispositifs prudentiels : en dépit de ces nécessaires évolutions, la Banque centrale reste au cœur du dispositif prudentiel. Le banquier central se situe ainsi au cœur du processus de construction des systèmes monétaires et bancaires de part sa mission d'émission de la monnaie et de conduite de la politique monétaire, mais aussi de son intervention in fine pour opérer le "bouclage » du système bancaire. En assurant normalement le refinancement ultime des établissements de second rang, tout comme en intervenant en sa qualité de prêteur en dernier ressort pour assurer la liquidité du marché monétaire et juguler la propagation d'une crise, il garantit la pérennisation des échanges monétaires.

En cela, la Banque centrale garantit l'intégrité du système de paiement (Aglietta, Cartelier, 1998), en assurant aux agents privés la pérennité du secteur bancaire dans sa mission d'affectation de l'épargne et de financement de l'économie, et aux autorités le bon fonctionnement du vecteur de transmission des impulsions de la politique monétaire : elle préserve la confiance dans la monnaie.

Au-delà des différentes stratégies nationales adoptées par les économies en transition d'Europe centrale (telles que la «thérapie de choc » en Pologne, le "gradualisme » en Hongrie et le «libéralisme » tchèque), la Banque centrale peut être analysée comme l'agent assurant le double ancrage de la monnaie, sous l'angle de la politique monétaire - en assurant la stabilité des prix - mais aussi sous celui de la politique prudentielle en prévenant le risque systémique. Dans le cadre de la construction de systèmes monétaires et bancaires, la Banque centrale pourra alors être considérée comme la « clef de voûte » du dispositif institutionnel, ce qui légitime son indépendance, tant visà-vis du gouvernement que de la sphère privée. Son degré d'indépendance, tant politique qu'économique, fournit ainsi un bon indicateur du niveau atteint par le processus d'institutionnalisation de la monnaie - dont elle est le gardien et le garant dans les économies en transition, c'est-à-dire, in fine, du degré d'achèvement du processus transformationnel dans la sphère monétaire et bancaire de ces pays. Conclusion 
31 L'analyse du processus de transition comme construction institutionnelle de la monnaie nous semble ainsi avoir le grand avantage de proposer une lecture plus qualitative de ce processus et du développement des pays d'Europe centrale.

Il apparaît clairement que la Banque centrale, garante de la stabilité du système monétaire par la préservation de la confiance des agents dans la monnaie - sous la double dimension de la stabilité de la monnaie et de la pérennisation du système de paiement - est la gardienne de l'institution monétaire, élément fondateur des économies de marché assurant la socialisation des agents. Son rôle est donc par nature institutionnel, ce qui signifie qu'il s'inscrit dans le processus de structuration du marché dont il émane :

la hiérarchisation du système s'opère par un phénomène d'auto-organisation des paiements dans la communauté bancaire [...] la création des banques centrales ne serait alors que l'aboutissement d'un processus évolutionnaire de marché (Scialom, 1999).

Néanmoins, l'indépendance de jure - dont le niveau apparaît satisfaisant pour les Banques centrales tchèque, polonaise et hongroise qui obtiennent le même indice que la Bundesbank dans les évaluations effectuées par W. Maliszewski (2000, p. 749-789) par exemple $e^{23}$ - apparaît comme étant loin d'être suffisante pour assurer la crédibilité du banquier central: les pressions auxquelles ont été soumises certaines banques centrales nouvellement indépendantes dans les économies en transition en sont une claire illustration. Elle peut n'être qu'illusoire sans l'indépendance de facto qui se construit progressivement en s'appuyant sur la confiance et la crédibilité que lui accordent les acteurs économiques. C'est par la défense de la monnaie en sa qualité de lien social que le banquier central acquiert progressivement la confiance des agents économiques dans un processus qui est bien celui d'une construction institutionnelle, et qui est, selon nous, encore à l'œuvre aujourd'hui dans les économies en transition d'Europe centrale. Warren Coats et Marko Skreb (2000) soulignent néanmoins les difficultés de ce processus :

Pour faire de la Banque centrale une institution respectable dans la création d'un consensus social autour de politiques judicieuses au-delà de simples questions bancaires, il faut parfois du temps, du courage, de la détermination, une influence politique et beaucoup de patience ${ }^{24}$.

\section{BIBLIOGRAPHIE}

Aglietta M., J. Cartelier, 1998, « Ordre monétaire des économies de marché », in Aglietta M., Orlean A. (eds), La monnaie souveraine, Paris, Odile Jacob.

Aglietta M., Orléan A., La monnaie entre violence et confiance, Paris, Odile Jacob, 2002.

Aoki M., 2001, Towards a Comparative Institutional Analysis, Cambridge, Ma, The MIT Press.

Arrow K. J., 1998, "The place of Institutions in the Economy : A Theoretical Perspective ", in Hayami Y. and M. Aoki (1998), The Institutional Foundations of East Asian Economic Development, London, MacMillan, p. 39-48. 
Blanchard O., 1997, The Economics of Postcommunist Transition, Oxford, Clarendon Press.

Boyer R., 2001, « La grande transformation russe comme analyseur de la pertinence des théories économiques ", contribution au Colloque franco-russe Les économistes face à dix années de transition en Russie, Collège universitaire français de Moscou, 1-2 juin, EHESS-CEMI.

Cartelier J., 1991, « Monnaie et système de paiement : le problème de la formation de l'équilibre », Revue Française d'Économie, vol. 6, n 3, été.

Chavance B., 2001, « Organisations, institutions, système : types et niveaux de règles », Revue d'économie industrielle, $\mathrm{n}^{\circ} 97,4^{\mathrm{e}}$ trim.

Chavance B., 2002, « Why National Trajectories of Postsocialist Transformation Differ? », EastWest, Dec.

Coats W. and M. Skreb, 2000, « Banques centrales et transition : vue d'ensemble des principaux problèmes après dix ans ", Revue d'Économie Financière, $\mathrm{n}^{\circ}$ Hors-Série Dix ans de transition en Europe de l'Est - Bilans et Perspectives, AEF, Paris.

Diamond D. W., 1984, « Financial Intermediation and Delegated Monitoring », Review of Economic Studies, $\mathrm{n}^{\circ} 51$.

Fama E., 1980, « Banking in the Theory of Finance », Journal of Monetary Economics, $\mathrm{n}^{\circ}$ 1, january.

Favereau O., 1989, « Marchés internes, marchés externes », Revue économique, vol. 40, n² 2, L'économie des conventions, mars.

Greif A., 1994, «Cultural Beliefs and Organization of Society : A Historical and Theoretical reflection on Collectivist and Individualist Societies », Journal of Political Economy, 102, p. 912-950.

Keynes J. M., 1935, (éd. française de 1979), Théorie générale de l'emploi, de l'intérêt et de la monnaie, Paris, Petite Bibliothèque Payot.

Kornaï J., 2001, « Le paradigme systémique », B. Chavance et M. Vahabi (ed.), La transformation économique postsocialiste, Paris, Maison des Sciences de l'Homme.

Kornaï J., 2000, Ten Years After "The Road to a Free Economy" : The Author's Self-Evaluation, Annual Bank Conference on Development Economics - ABCDE, April 18-20, World Bank.

Kornaï J., 1984, Socialisme et économie de la pénurie, Paris, Economica.

Kreps D. M., 1990, A Course in Microeconomics Theory, London, McGrawHill ed.

Maliszewski W., 2000, "Central bank independance in transition economies », Economics of Transition, vol. 8 (3), EBRD, UK, Blackwell Publishers, p. 749-789.

Miotti L. et Plihon D., 2001, « Libéralisation financière, spéculation et crises bancaires ", Revue Internationale, $\mathrm{n}^{\circ} 85(1), 1^{\mathrm{er}}$ trimestre, CEPII.

Nelson R., 1994, « The Co-evolution of Technology, Industrial Structure and Supporting Institutions ", Industrial and Corporate Change, 3.

North D., 1994, « Institutional Change: A Framework of Analysis », Economics Working Papers, Economics Department of Washington University (disponible sur la toile en mars 2006 : http:// ideas.uqam.ca/ideas/data/wpawuwpeh.html)

North D., 1997, The Contribution of the New Institutional Economics to an Understanding of the Transition Problem, WIDER Annual Lectures, United Nations University/World Institute for Development Economics Research, Washington. 
Ostroy J. M. et R. Starr, 1990, « The transaction role of money », ed. by B. R. Friedman et F. H. Hahn, Handbook of Monetary Economics, ch. 1, vol. 1, Elsevier science publishers B.V.

Renversez F., 1996, « Money Circulation and Overdraft Economy » in Deleplace et E. Nell (ed.), Money in Motion - The Postkeynesian and circulation approaches, Mac Millan.

Renversez F., 2001, « La transition inaboutie du système financier en Russie », Diogène, n 194, Recomposition des espaces postcommunistes, Paris, avril-juin, Unesco.

Sapir J., 1989, L'économie mobilisée, Paris, La découverte.

Scialom L. et Y. Zlotowski, 1994, « Les fondements institutionnels de la crise monétaire russe, un éclairage théorique ", Revue d'économie politique, nº 104 (5), sept.-oct.

Smith A., 1759, Théorie des Sentiments moraux.

Stintzy O., 2003, « La transition monétaire en Europe centrale : une lecture institutionnelle », Actes du Colloque L'ouverture de l'Europe à l'Est, Université de Tours, 23, 24 et 25 janvier.

Stintzy O., 2004, Une lecture institutionnelle de la construction de systèmes monétaires et bancaires dans les économies en transition d'Europe centrale: Hongrie, Pologne, République tchèque, Thèse pour le doctorat en sciences économiques, sous la direction de Madame Françoise Renversez, université Paris X-Nanterre.

Zlotowski Y., 1998, La monnaie dans la transition russe (1992-1997), Thèse de doctorat en sciences économiques, sous la direction de Monsieur le professeur Michel Aglietta, université de Paris XNanterre.

\section{NOTES}

1. Une version plus détaillée de ce texte a été publiée sous forme de document de travail par le laboratoire EconomiX, université de Paris X-Nanterre (http:// economix.uparis10.fr/fr/axes/mmei/membres/). L'auteur remercie Mme le professeur F. Renversez pour l'aide précieuse apportée pour la rédaction de ce texte.

2. J. Kornaï, 2001.

3. Voir aussi O. Stintzy, 2004.

4. «Une économie peut globalement être considérée comme un groupe - ou un "bouquet" - robuste et cohérent d'institutions mutuellement interdépendantes » (traduit par l'auteur), M. Aoki, 2001.

5. Ou construction institutionnelle de la monnaie.

6. Cette faiblesse (ou absence) de contrainte budgétaire ne s'applique cependant qu'au secteur productif, alors que seul le circuit fiduciaire pouvait être utilisé par les particuliers et les ménages.

7. Cette régularisation a été effectuée selon une triple procédure d'enregistrement comptable centralisé (crédit bancaire équivalent accordé à l'entreprise victime de nonpaiement), de refinancement automatique des banques en fonction du volume de crédit accordé hors-plan et enfin d'annulation des dettes mutuelles entre entreprises (J. Sapir, 1989).

8. Schématiquement, une première phase est représentée par le processus de tâtonnement effectué par le commissaire-priseur walrassien qui, en modifiant les prix, cherche à faire coïncider l'offre et la demande en fonction des quantités proposées et demandées par chacun des agents ; ce n'est qu'une fois qu'un accord est trouvé sur un 
prix d'équilibre que le commissaire-priseur communique celui-ci à l'ensemble des participants au marché, ce qui les « autorise " à réaliser effectivement les transactions (J. Cartelier, 1991).

9. De cette impossibilité de connaître a priori les conditions de réalisation des différentes transactions entreprises découle logiquement un risque de violation de la contrainte budgétaire des agents : ceux-ci doivent donc être considérés comme présentant une rationalité limitée par la nature même du mode de coordination par le marché : en effet, l'hypothèse d'anticipations rationnelles priverait ici le marché de toute signification puisque des agents qui connaîtraient a priori les conditions optimales de réalisation de leurs échanges n'auraient aucun besoin d'un instrument de coordination pour les réaliser.

10. Dans la théorie orthodoxe, c'est l'équilibre qui représentait la modalité d'accord entre les agents, celui-ci décrivant la meilleure solution pour chacun d'entre eux compte tenu des trajectoires des autres participants.

11. Ceci afin d'éviter les phénomènes de «tricheries » sur les excès d'offre et de demande réciproques : en effet, étant seul connaisseur de ses excès d'offre et de demande, chaque acteur peut prétendre, vis-à-vis de n'importe quel autre participant au marché, avoir une demande insatisfaite résultant d'un précédent échange déséquilibré (avec un tiers) et lui demander de la satisfaire afin de voir son équilibre budgétaire rétabli, et ceci au nom du rétablissement de l'équilibre général.

12. En effet, la détention monétaire suppose soit sa disponibilité en caisse, soit la réalisation d'actifs préalablement acquis, soit, enfin, l'accès au crédit. Ce dernier point est important car il indique une prise de décision favorable de la part d'un intermédiaire financier bénéficiaire d'un avantage informationnel qui peut provenir de la fonction de «teneur de livres » pour son client, comme l'a mis en évidence E. Fama (1980) ; cet avantage peut aussi provenir d'un investissement informationnel effectué par l'intermédiaire financier basé lui-même, en partie, sur les « scores » passés de l'emprunteur comme l'a souligné D. W. Diamond (1984).

13. J. Cartelier, 1991, souligné par nous.

14. A. Smith, Théorie des sentiments moraux, 1759, cité par M. Aoki, 2001 et traduit par l'auteur.

15. «A self-sustaining system of shared beliefs about a salient way in which the game is repeatedly played », M. Aoki, 2001 (traduit par l'auteur).

16. M. Aoki, 2003: " a salient feature of an equilibrium may be tacitely recognized by the agents, or have corresponding symbolic representations outside the minds of agents and coordinate their beliefs » [une caractéristique particulière d'un équilibre (du jeu) peut être tacitement reconnue par les agents, ou avoir des représentations symboliques correspondantes en dehors de l'esprit des agents et coordonner leurs croyances] (traduit par l'auteur).

17. De la même manière, explique $\mathrm{M}$. Aoki, que les prix relatifs sont des informations suffisantes pour effectuer des transactions dans l'économie de marché, sans que les agents aient nécessairement à connaître l'ensemble des caractéristiques de l'environnement.

18. «Portions of agents equilibrium beliefs common to (almost) all of them regarding how the game is actually played ", M. Aoki, 2001.

19. D. M. Kreps, 1990 [Une institution est le produit des expériences de long terme d'une société composée d'individus présentant une rationalité limitée et un regard rétrospectif.] (traduit par l'auteur). 
20. F. Renversez, 2001.

21. J. Kornaï, souligne ainsi que « pour environ un quart ou même un tiers de la population mondiale, le changement de système a été une expérience cathartique : ceux qui vivent maintenant dans la partie du monde en transformation ne peuvent pas et ne continueront pas à vivre comme ils l'ont fait auparavant ", in B. M. Chavance, M. Vahabi (ed.), 2001.

22. M. Aoki, 2001 (traduit par l'auteur).

23. Ce point est plus particulièrement développé dans O. Stintzy (2003), ainsi que dans la version plus détaillée de ce texte publiée sous forme de document de travail par le Modem, université de Paris X-Nanterre.

24. Les auteurs utilisent ici le terme " institution » dans le sens d'une " organisation », ce que marque bien la distinction entre « institution » et « institution respectable dans la création d'un consensus social », ce dernier terme correspondant bien à notre conception d'une institution (cf. M. Aoki, 2001).

\section{RÉSUMÉS}

Olivier Stintzy. Analyse néo-institutionnaliste du processus de transition monétaire en Europe centrale Dans une économie de marché, la monnaie est une institution qui rend possible la coordination décentralisée des agents. Ainsi, la transition économique en Europe centrale et orientale (République tchèque, Hongrie, Pologne) peut-elle être analysée en termes de construction d'une institution monétaire. La politique monétaire a pour but de forger une stabilité monétaire en introduisant des réformes par séquences dont la variable d'ajustement est le taux de change. Dans les trois pays d'Europe centrale considérés, l'ultime étape de cette politique monétaire est la mise en place du système de ciblage de l'inflation. Des progrès impressionnants ont été réalisés dans ces pays depuis la fin de l'économie planifiée. Cependant, les traits d'une faiblesse résiduelle importante montrent que le processus de transition, d'un point de vue institutionnel, n'est pas encore achevé. La banque centrale, institution indépendante et garantie de stabilité, reste donc au cœur du processus qui conditionne la confiance que les agents peuvent avoir dans la monnaie.

Olivier Stinzy. A neo-institutionalist approach to monetary transition in Central Europe

In a market economy, money is the social institution that enables the coordination of agents' decentralised actions. Therefore, the economic transition in Central Europe (Czech Republic, Hungary, Poland) can be analysed as the building of the monetary institution. The monetary policy aims at forging monetary stability by following a sequence of reforms whose adjustment variable is the exchange rate. In the three Central European countries considered, the ultimate stage of this monetary policy is an inflation targeting system. Impressive progresses have been made in these countries since the end of the planned economy. However, the remaining important weaknesses indicate that the transition process, in its institutional dimension, has yet to be fully achieved. The independent Central Bank - guarantor of money stability - therefore resides at the heart of the process which preserves the agents' confidence in money. 
AUTEUR

OLIVIER STINTZY

Docteur en sc. économiques. Chercheur associé à EconomiX, université Paris 10-

Nanterre, olivierst@gmail.com 